\title{
Fra-1 regulates inflammatory and degenerative arthritis
}

\author{
T Kireva', K Zwerina', W Baum', S Hayer², G Schett ${ }^{1}$, J Zwerina ${ }^{1 *}$ \\ From 5th European Workshop on Immune-Mediated Inflammatory Diseases \\ Sitges-Barcelona, Spain. 1-3 December 2010
}

\section{Introduction}

Rheumatoid arthritis (RA) and osteoarthritis (OA) are chronic joint diseases that target synovial membrane, cartilage and bone. Cartilage and bone damage is a hallmark of both diseases and often necessitates joint replacement surgery during the disease course.

\section{Aim}

To evaluate the impact of chronic Fra-1 over-expression on experimental models of inflammatory and degenerative arthritis.

\section{Methods}

Fra-1tg mice were crossed with hTNF-transgenic (hTNFtg) mice. Clinical and histological signs of arthritis, synovitis and cartilage damage were established. In situ hybridization (ISH) for osteocalcin and osteopontin were performed. Structural and dynamic parameters of systemic bone were evaluated by bone histomorphometry and calcein labelling. In vitro studies with wt and fra-1tg chondrocytes were performed for gene expression.

Further, an established OA model (collagenaseinduced OA) was performed in wildtype and fra-1 $\mathrm{tg}$ mice. Cartilage degeneration and osteophyte formation were evaluated.

\section{Results}

Mice overexpressing Fra-1 and hTNF showed increased clinical arthritis scores as compared to hTNFtg mice. Histological analysis showed an increase of synovial inflammation in Fra-1tgxhTNFtg mice. These mice had virtually no synovial bone erosions and showed enhanced osteoblast function and matrix deposition compared to hTNFtg mice. Interestingly, cartilage integrity was also preserved in
Fra-1tgxhTNFtg mice. In vitro TNF-stimulated fra-1tg chondrocytes showed reduced expression of matrix metalloproteinases as compared to wildtype chondrocytes challenged with TNF. Further, fra-1tg chondrocytes showed higher proliferative rates and expression of cyclin genes compared to wildtype chondrocytes.

Finally, preliminary results suggest a cartilage-protective effect of Fra-1 in the collagenase-model of OA.

\section{Discussion}

This study shows that increased osteoblastic function is able to prevent local and systemic bone loss in arthritis despite active signs of local and systemic inflammation. Further, overexpression of the transcription factor Fra-1 seems to reduce cartilage destruction in hTNFtg mice and an experimental OA model. Strategies to build up bone and protect cartilage might therefore be considered as key tools to preserve joint architecture.

\section{Author details}

'Dept. of Internal Medicine 3, Immunology and Rheumatology, University of Erlangen, Germany. ${ }^{2}$ Dept. of Internal Medicine 3, Division of Rheumatology, Medical University of Vienna, Austria.

Published: 25 November 2010 